\title{
Matrix regeneration agents improve wound healing in non-stressed human corneal epithelial cells
}

A Robciuc ${ }^{1}$, RPJ Arvola', M Jauhiainen ${ }^{2}$ and JM Holopainen ${ }^{1,3}$

\begin{abstract}
Purpose Matrix regenerating agents (RGTAs) emerged as promising in vivo wound-healing agents. These agents could prove beneficial for the treatment of dry eye diseaseassociated corneal micro-erosions; therefore, we aimed to evaluate the wound healing efficacy of regenerative agents (RGTAs or serum) in an in vitro model of hyperosmolarity (HO) stressed and nonstressed human corneal epithelial cells. Patients and methods The migration and proliferation induced by the regenerative agents was evaluated using an in vitro scratch wound assay and brome-deoxy-uridine incorporation. The inflammatory profile and effects of osmoregulators were also investigated. The two-tailed paired $t$-test calculated the statistical significance, with $P$-value $<0.05$ considered significant.

Results The most efficient inducer of reepithelization was $2 \%$ serum, followed closely by $2 \%$ RGTA with an average improvement in cell migration of 1.8- and 1.4-fold, respectively, when compared with the non-treated control. Hyperosmolar stress significantly reduced the restorative effects of both serum and RGTAs; these effects were, however, neutralized by the osmoregulator betaine.

Conclusion These findings suggest that RGTAs could provide efficient treatment for dry-eye associated corneal micro-lesions if ocular surface $\mathrm{HO}$ is neutralized.

Eye (2018) 32, 813-819; doi:10.1038/eye.2017.277; published online 22 December 2017
\end{abstract}

\section{Introduction}

The corneal epithelial wound-healing response is the result of a cascade of events involving enzymatic and cytokine-mediated interactions between the cells at the ocular surface and corneal nerves. ${ }^{1}$ This orchestrated rescue response can efficiently restore corneal structure and function in most situations. Dry eye disease (DED) is associated with increased tear fluidosmolarity, which affects the efficiency of the wound-healing process. ${ }^{2}$

Treatment of DED ranges from the use of lubricants and lid hygiene to anti-inflammatory therapies, autologous serum treatment ${ }^{3}$ or amniotic membrane transplantation. ${ }^{4}$ Autologous serum therapy has produced promising results, similar to tears, serum has similar $\mathrm{pH}$ and osmolarity along with nutritional factors, which will maintain cellular viability in the repair processes and bactericidal components to further reduce the risk of contamination and infection. ${ }^{3}$ Encouraging results for the treatment of epithelial erosions and corneal ulcers have been reported very recently from the use of matrix regenerating agents (RGTAs). ${ }^{5,6}$ RGTAs are heparan-sulphate mimetic polymers that are resistant to enzymatic cleavage and that were shown to induce extracellular matrix remodeling by decreasing the production of collagen III and modulation of the protease activity. ${ }^{7,8}$ These actions converge towards an improved in vivo speed and quality of tissue repair tested in several experimental models. ${ }^{9}$ This is accomplished by providing a scaffold for the cells and by harboring the growth factors, cytokines, or chemokines with affinity for heparin/heparan sulphates. It remains unknown, however, whether commercial RGTAs stimulate the migration and/or proliferation of the epithelial cells, or if their action is sensitive to micro-environmental factors at the ocular surface. Consequently, our study sought to examine how RGTA performs in a simple in vitro wound-healing model of corneal epithelial cells, when compared with serum treatment. To test the efficacy of such treatment in dry eye-associated lesions, we measured the wound-healing capacity of the above-mentioned

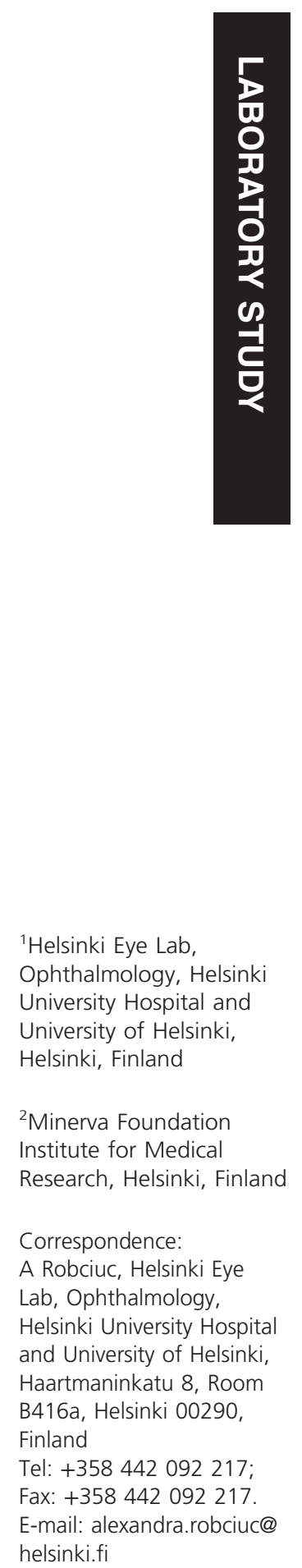

${ }^{3}$ Professor Juha Holopainen passed away after all aspects of this study were finalized.

Received: 7 April 2017

Accepted in revised form: 29 October 2017

Published online:

22 December 2017 
agents under hyperosmolar conditions in the presence or absence of osmoregulators.

\section{Materials and methods}

\section{Cell culture}

SV-40 immortalized human corneal epithelial cells (HCE cells) ${ }^{10}$ were grown and maintained in DMEM/F12 supplemented with 15\% fetal bovine serum (FBS) and EGF, insulin, gentamicin (all from Thermo-Fisher Scientific, Waltham, MA, USA) and cholera toxin (Sigma, St. Louis, MO, USA). The initial cell batch was characterized for specific markers and expression profile. ${ }^{11,12}$ The cells were kept in culture for 8-10 passages after defrosting and their appearance was constantly monitored by light microscopy. Before experiments, the cells were serum starved for $18 \mathrm{~h}$. The RGTA (Cacicol, Laboratoires Thea, Clermont-Ferrand, France) and serum were added to the serum-free media in $2 \%$ final concentration. For the scrape wound assay the monolayer was washed once with warm PBS and then the scrape wound was created using a sterile plastic pipette tip. The cells removed in the scraping were washed away with PBS and the cells were incubated for a determined time in the presence and absence of the wound-healing promoting agents. Hyperosmolarity $(\mathrm{HO})$ was induced through the addition of $\mathrm{NaCl} 5 \mathrm{M}$ to the cell culture medium. Osmoregulators (betaine, L-carnitine, and erythritol, Sigma) were added to the medium with or without regenerative agents one hour before the increase in osmolarity.

\section{Scratch wound assay}

The cell monolayer was scraped, as mentioned above, in a straight line with a pipet tip and debris was removed. ${ }^{13}$ To create reference points for image acquisition the plate bottom was marked with a line perpendicular to the scratch mark. Images were taken immediately after the scrape wound was made $\left(t_{0}\right)$ right above and below the line using a phase-contrast microscope and $\times 10$ magnification (Leica DMIL microscope, Wetzlar, Germany). After incubation images were taken using the same reference points. Four images were acquired for each well. Each experiment was repeated in triplicates at least 3 times. The images were analyzed using the ImageJ software (NIH free software v1.42q, USA) on non-altered original files. The cell-free area was measured at $t_{0}\left(\mathrm{At}_{0}\right)$ and then after incubation ( $\mathrm{At}_{1-48}, t_{1-48}$ is the incubation time range in hours) and results are shown normalized to $t_{0}$. Re-epithelization rate was defined as $A t_{0}-A_{18} / A_{0}$. To simplify comparison, the re-epithelization rate of control cells was set to 1 .

\section{Cell proliferation assay}

Cell proliferation stimulated by the regenerative agents was measured using a colorimetric bromodeoxyuridine (BrdU) incorporation assay (Roche Diagnostics,

Mannheim, Germany) following the manufactures' guide lines. Briefly, $1 \times 10^{4}$ cells were plated on a 96-well plate and serum-starved overnight. The cells were then incubated for $24 \mathrm{~h}$ in the presence of the regenerative agents with or without increased osmolarity. The samples were labelled with the BrdU for $2 \mathrm{~h}$. After fixation and denaturation, samples were incubated 90 min with the anti-BrdU-POD antibody. The POD substrate was incubated for $5 \mathrm{~min}$ after which the reaction was stopped with $1 \mathrm{M}$ sulfuric acid and measured at $450 \mathrm{~nm}$ using a multimode plate reader EnSpire (Perkin-Elmer, Waltham, MA, USA). The experiments were performed in duplicates and replicated four times.

\section{Cytokine assays}

IL-8 secretion was measured from cell-conditioned media using a DuoSet ELISA for human CXCL8/IL-8 (R\&D Systems, Minneapolis, MN, USA) and as recommended by the manufacturer. Samples were diluted 1:1 (vol/vol) with Reagent Diluent. Plates were analyzed using the multimode plate reader EnSpire (Perkin-Elmer) at $450 \mathrm{~nm}$. Each measurement was performed in triplicates and repeated three times. Cytokine/chemokine secretion in HCE medium was quantified using the Proteome Profiler Array-Human Cytokine Array Panel A (R\&D Systems) with 36 different cytokines and chemokines spotted in duplicates on the nitrocellulose paper. Cell-conditioned medium $(700 \mu \mathrm{l})$, cleared of cell remnants and debris, was mixed with the kit reagents, and incubated with the arrays as instructed in the assay protocol. Bound cytokines were identified using chemiluminescent detection reagents.

\section{Statistical analysis}

Data are presented as mean values from three independent experiments \pm SEM, unless otherwise specified. The measured values were considered with normal distribution and of equal variance and were evaluated by two-tailed paired $t$-test. A $P$-value $<0.05$ was considered significant.

\section{Results}

RGTAs stimulates cellular migration in an in vitro model of the corneal epithelium

As there are no previous data on an in vitro translation of the RGTA dosage, we first determined the optimal 

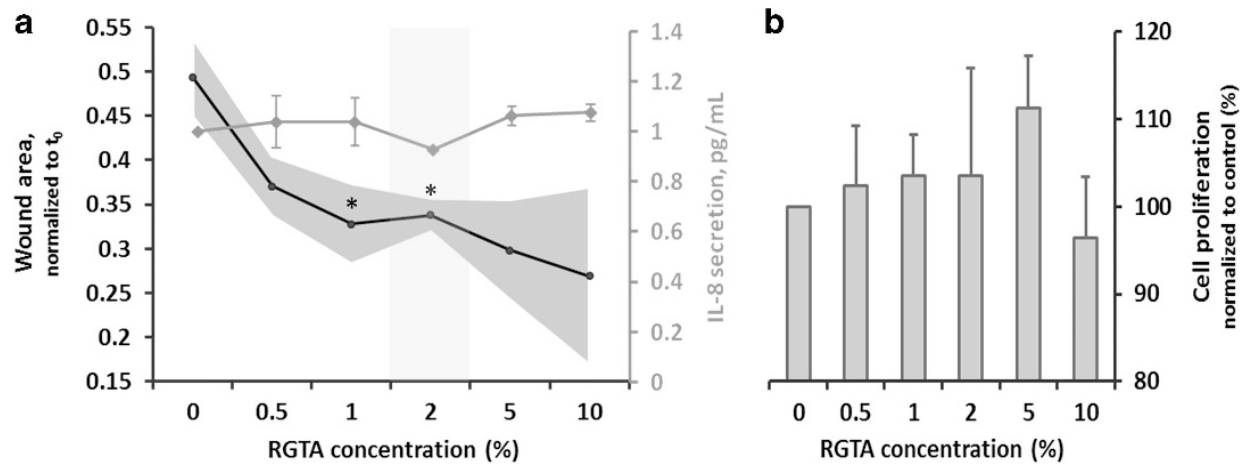

Figure 1 Cacicol effects on cell migration and proliferation in an in vitro model of the corneal epithelium. (a) Cell migration assay. After the scrape wound was made HCE cells were incubated for $18 \mathrm{~h}$ in the presence of $0.5-10 \%$ RGTA (Cacicol, vol/vol). The black line represents the healing rate normalized to the $t_{0}$ of the scratch wound. The grey area that follows the black line denotes the SEM intervals. The grey line presents the IL- 8 concentration in the medium with the values on the secondary $Y$ axis. ${ }^{*} P<0.05$ when compared with the non-treated control. (b) Proliferation assay. HCE cells were incubated with increasing RGTA concentration for $24 \mathrm{~h}$ and then BrdU incorporation was measured using an ELISA assay.

concentration of the RGTA to be used in the cell culture medium. After the scrape wound was made, the cells were incubated for $18 \mathrm{~h}$ in the presence of RGTAs ranging from 0 to $10 \%$ (vol/vol, Figure 1). Even low levels of RGTA in the media improved the re-epithelization of the model wound by $30 \%$ compared with control cells, values reaching statistical significance at dose levels of 1 and $2 \%$. A cell proliferation assay demonstrated that the RGTA can slightly enhance the proliferation of the HCE cells ( $10 \%$ increase in the BrdU incorporation for $0.5-5 \%$; Figure 1b). Because of the similar RGTA/cell surface ratio between our in vitro model and the clinical setting (roughly, one drop of RGTA per $\mathrm{cm}^{2}$ of corneal epithelial cells), we chose the $2 \%$ RGTA concentration for further investigations. Figure 1a additionally shows that IL-8 (an acute stress biomarker) secretion from the HCEs maintains normal levels regardless of the concentration of the RGTA in the medium.

We then compared the effect of serum and RGTA on cell migration speed in a corneal wound-healing model. The FBS as well as the RGTA were diluted to $2 \%$ in the culture medium. As expected, $2 \%$ serum was the most effective promoter of migration (healing rate $=1.8$, Figures $2 \mathrm{a}$ and $\mathrm{b}$ ). Incubation with RGTA produced comparable results, with a re-epithelization rate of 1.4 when compared with control. Moreover, the reepithelization speed over a $48 \mathrm{~h}$ period was similar between serum and RGTA, with the former showing a slightly faster rate between 10 and $20 \mathrm{~h}$ from wounding (Figure 2c).

\section{Hyperosmolar stress significantly affects the RGTAs efficiency}

DED is associated with increased tear osmolarity known to delay wound healing, ${ }^{2}$ but it is not known whether
HO similarly hinders the RGTA-promoted healing. Therefore, we tested the effect of increasing osmolar stress on the re-epithelization rate of the scratch wounds in the presence of RGTA or FBS. The starting point was the normosmolar culture media (309 mOsm and salts $140 \mathrm{~mm}$ ) and the osmolarity was gradually increased by adding $\mathrm{NaCl} 5 \mathrm{M}$ solution. As observed in vivo, $\mathrm{HO}$ significantly attenuated the healing process of the scrape wound; moreover, increasing the salt concentration with $100 \mathrm{mM}$ or more resulted in an increase in the size of the scrape wound displaying cytotoxicity with no improvement from the addition of either RGTA or serum (Figure 3a).

We have previously shown that $\mathrm{HO}$ induces significant secretion of the pro-inflammatory cytokine IL-8 from the HCE cells. ${ }^{14}$ Neither RGTA nor FBS prevented or diminished the secretion of IL-8 from HCEs (Figures $3 b$ and c). Similar effects were measured for other HCE-secreted cytokines ${ }^{14}$ (such as macrophage migration inhibiting factor, IL-1 receptor antagonist, SerpinE1, IL-6 or GRO-alpha-data not shown).

\section{$B r d U$ incorporation in the presence of regenerative agents}

Both regenerative agents tested stimulated cell proliferation with 10-20\% when compared with control, under non-stressed conditions (Figure 4). The increase in osmolarity by the addition of $\mathrm{NaCl}$ significantly affected the viability of the cells as demonstrated by the decrease in BrdU incorporation. FBS and RGTA, however, retained their minute positive effect on proliferation even during mild $\mathrm{HO}$ (Figure 4). 
a

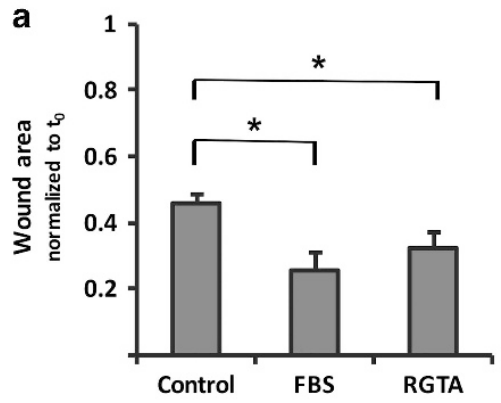

b

$t_{0}$
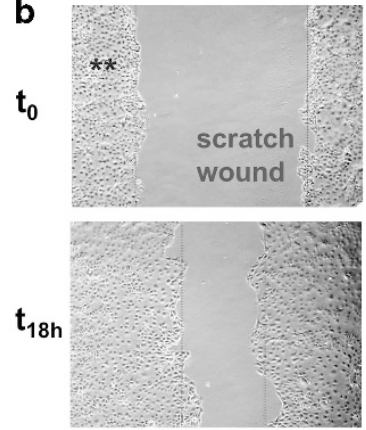

control

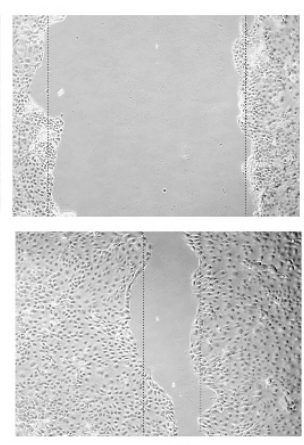

FBS

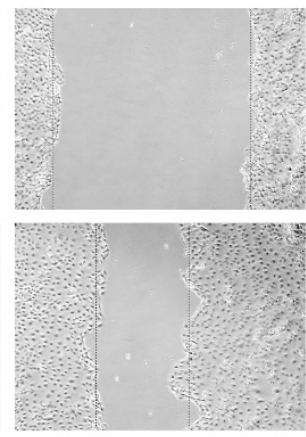

RGTA

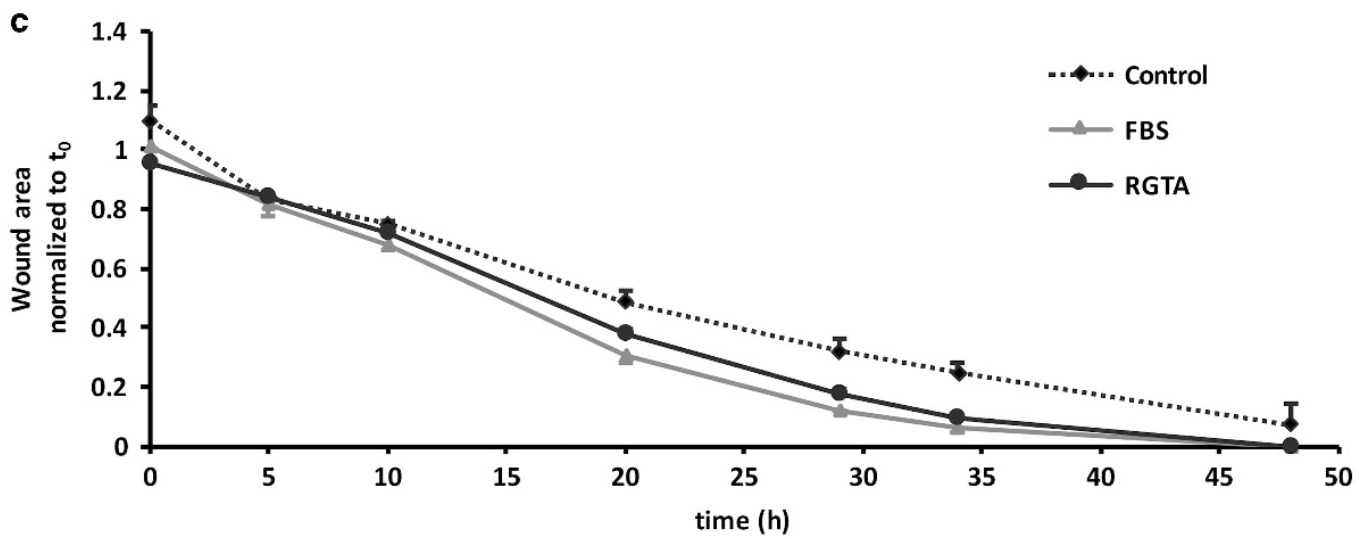

Figure 2 Comparison between RGTA (Cacicol) and serum in their ability to induce corneal epithelial cell migration. (a) Wound healing rates for $2 \%$ RGTA (Cacicol) and 2\% serum (FBS) after $18 \mathrm{~h}$ of incubation. (b) Representative images of the scrape wound at $t_{0}$ and $t_{18 \mathrm{~h}}$. (c) Healing rate as a function of time. Images were recorded at 0, 5, 10, 20, 30, 34, and $48 \mathrm{~h}$. Images were acquired with a digital camera mounted on a phase-contrast microscope with $\times 10$ magnification. ${ }^{*} P<0.05$; ${ }^{* *}$ corneal epithelial cell monolayer.

a

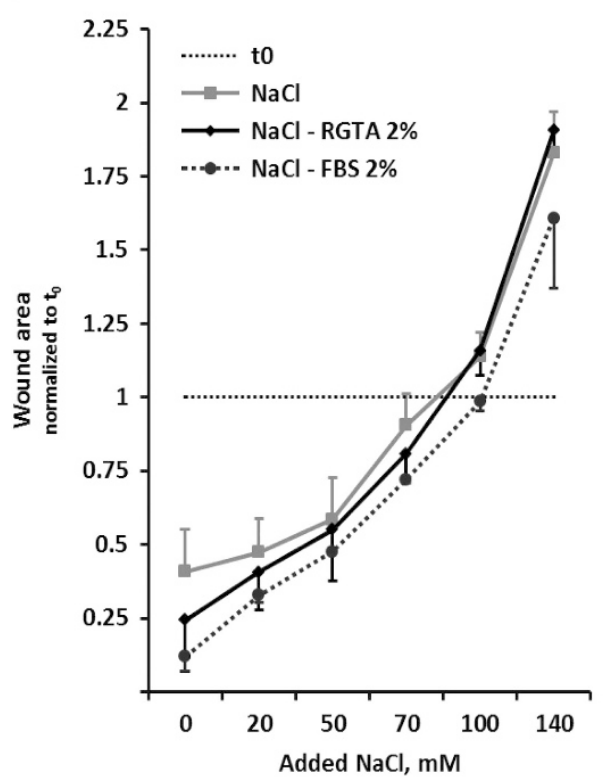

b
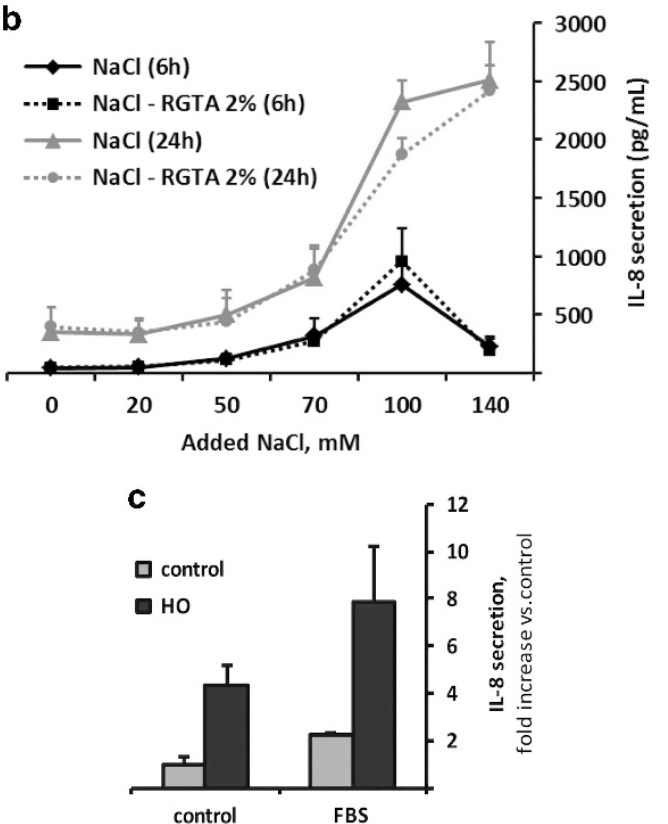

Figure 3 Effects of RGTA and serum on wound healing under hyperosmolar stress conditions. (a) Wound-healing rate after $18 \mathrm{~h}$ incubation with increasing concentrations of $\mathrm{NaCl}$, in the presence or absence of RGTA (Cacicol) or FBS. (b) IL- 8 secretion at 6 and $24 \mathrm{~h}$ after the addition of $\mathrm{NaCl}$. (c) IL-8 secretion after $18 \mathrm{~h}$ in the presence of FBS compared with control with/without HO stress. HO was attained by the addition $\mathrm{NaCl}$ to a final concentration of $240 \mathrm{~mm}$. 


\section{Effects of osmoprotectants on HO cytotoxicity and} RGTAs

Osmoprotectants such as L-carnitine, erithritol, or betaine have been shown to significantly reduce HO-induced proinflammatory responses, ${ }^{15}$ to promote the survival of corneal epithelial cells, and protect the integrity of the epithelium. ${ }^{16-18}$ We therefore tested the effects of these molecules on wound healing under HO conditions (Figure 5). Although L-carnitine and erithritol proved ineffective (Figures $5 \mathrm{a}$ and $\mathrm{c}$ ), betaine showed a concentration-dependent improvement in $\mathrm{HO}$ wound healing (Figure $5 \mathrm{~b}$ ). The addition of RGTA 2\% enhanced the effects of 5 and $10 \mathrm{~mm}$ betaine. RGTAs in conjunction with betaine eliminated the $\mathrm{HO}$ cytotoxic effects and restored the wound healing capacity of the HCE cells to control levels (Figure 5b).

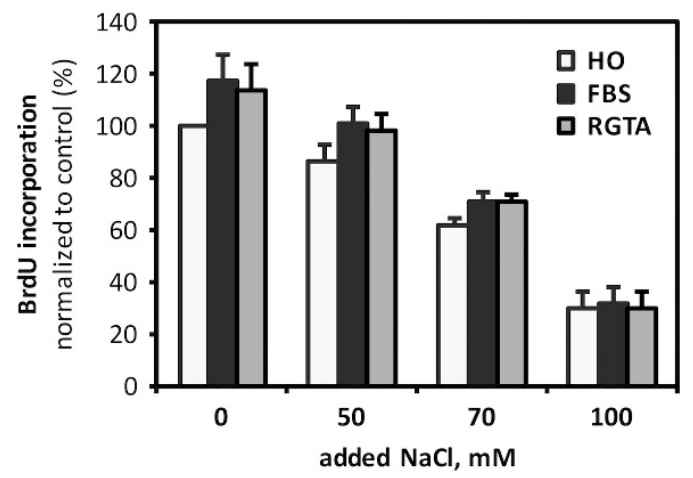

Figure 4 Proliferation induced by RGTA (Cacicol) or serum in HO-stressed and non-stressed corneal epithelial cells. Cells incubated with FBS $2 \%$ or RGTA $2 \%$ for $24 \mathrm{~h}$ compared with the non-treated control; $\mathrm{NaCl}$ concentrations display added $\mathrm{NaCl} .{ }^{*} \mathrm{P}<0.05$ compared with non-treated control. $\bullet P<0.05$ when compared with the RGTA/FBS control. Error bars indicate SEM values.

\section{Discussion}

Several studies have shown RGTA to be an efficient inducer of wound healing in corneal erosions and ulcers in clinical setting. ${ }^{5,6,19,20}$ We intended to obtain more basic data on the effects of RGTA using an in vitro wound healing model of corneal epithelial cells. What we could conclude is that RGTA is indeed an efficient promoter of healing in vitro and it almost matches the effects of serum, under non-stressed conditions, at the same concentration range. This is noteworthy considering that the RGTA only consists of a simple water solution of heparin-sulfate-like polymers with dextran and $\mathrm{NaCl}$, while fetal bovine serum is a complex mixture of crucial growth factors able to maintain cellular viability in the repair process. The dosing recommended in clinical practice proved to be fitting for cell culture conditions as well. Our model showed that RGTA had positive effects on cellular migration in the scrape wound healing even at very low concentrations $(0.5 \%)$, while concentrations higher than $2 \%$ did not bring significant further improvements. This also supports the idea that more frequent instillations of the ophthalmic solution will not improve the outcome. ${ }^{5}$

The positive results on wound healing from clinical trials $5,6,19,20$ encouraged us to postulate that RGTAs could be used to maintain the epithelial homeostasis in patients with DED. The results of our preliminary clinical tests have contradicted this hypothesis (unpublished data). Consequently, we tested whether the pre-existence of $\mathrm{HO}$ stress at the ocular surface would affect the efficacy of the RGTA treatment. We therefore monitored the repair of the scratch wound in increasing $\mathrm{HO}$ stress, in the presence or absence of the RGTA. What we could observe was that even small increments in the osmolarity of the medium had dramatic effects on the migration rate and proliferation of the HCE cells. The presence of FBS and

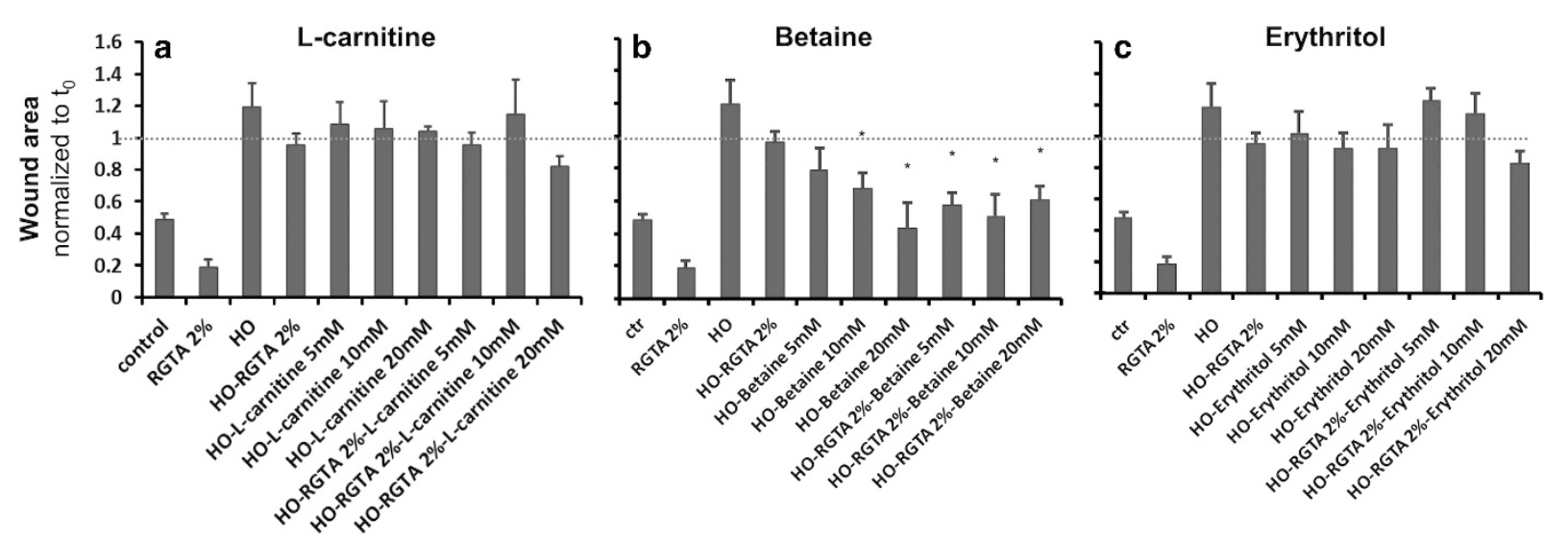

Figure 5 Effects of osmoprotectants on $\mathrm{HO}$ wound healing. The figure presents the wound-healing rate after $18 \mathrm{~h}$ of incubation. The punctate line denotes the wound area at $t_{0}$. L-carnitine (a), betaine (b), and erithritol (c) were added in the cell culture medium at the displayed concentrations $1 \mathrm{~h}$ before the addition of the RGTA and HO stimulation. ${ }^{*} P$-values $<0.05$ when compared with the HO control. 
RGTAs offered a modest benefit, when compared to control, but could not prevent the HO cytotoxic effects on the HCE cells. Moreover, none of the regenerative agents could prevent the secretion of the stress cytokine IL- 8 , the major neutrophil attractant in vivo. The addition of osmoprotector molecules, such as betaine, abolished the negative effects of $\mathrm{HO}$, however, and returned the wound-healing capacity in the presence of RGTAs to control levels.

A limitation of our study is the use of an immortalized cell line with inherent resistance and capacity of proliferation in optimal conditions. This advantage is not so evident in serum-starved conditions, however. The measured proliferation and migration could both be modulated by the addition of the regenerative agents and this supports the validity of our data. Moreover, preliminary data from a limited set of DED patients that received RGTAs confirmed the inefficiency of the medical device in cytotoxic conditions, such as increased osmolarity (data not shown).

Our results suggest that RGTAs are a promising therapy for wound healing at the ocular surface. A very recent study indicates that RGTAs could prove extremely useful for basement membrane dystrophy patients undergoing phototherapeutic treatment, ${ }^{21}$ for example. Pre-existing cellular stress attenuates their function, however, and delays the wound healing process. Clinicians should take into consideration that ocular surface micro-environment influences RGTA's efficacy and efficiency. Discontinuation of cytotoxic drugs should be considered when treating serious corneal wounds. ${ }^{20}$ Additionally, for dry eye patients, prior osmoprotection would convert the RGTA into beneficial healing inducers for, but not restricted to, DED associated lesions.

\section{Summary}

What was known before

- Matrix regenerating agents (RGTAs) emerged as promising in vivo wound healing agents.

\section{What this study adds}

- Our results suggest that RGTAs are a promising therapy for wound healing at the ocular surface. Pre-existing cellular stress attenuates their function, however, and delays the wound healing process. Osmoprotection converted the RGTAs into efficient healing inducers even under hyperosmolar conditions, suggesting that this therapy could be used for treating micro-lesions associated with dry eye.

\section{Conflict of interest}

The authors declare no conflict of interest.

\section{Acknowledgements}

This work was supported by grants from the Sigrid Juselius Foundation (JMH), the Helsinki University Central Hospital Research Foundation (JMH), and the Finnish Eye Foundation (JMH).

\section{References}

1 Lu L, Reinach PS, Kao WW. Corneal epithelial wound healing. Exp Biol Med (Maywood) 2001; 226: 653-664.

2 Bian F, Pelegrino FS, Pflugfelder SC, Volpe EA, Li DQ, de Paiva CS. Desiccating Stress-Induced MMP Production and Activity Worsens Wound Healing in Alkali-Burned Corneas. Invest Ophthalmol Vis Sci 2015; 56: 4908-4918.

3 Quinto GG, Campos M, Behrens A. Autologous serum for ocular surface diseases. Arq Bras Oftalmol 2008; 71: 47-54.

4 Muraine M, Gueudry J, Toubeau D, Gardea E, Verspyck E, Menguy E et al. Advantages of amniotic membrane transplantation in eye surface diseases. J Fr Ophtalmol 2006; 29: 1070-1083.

5 Aifa A, Gueudry J, Portmann A, Delcampe A, Muraine M. Topical treatment with a new matrix therapy agent (RGTA) for the treatment of corneal neurotrophic ulcers. Invest Ophthalmol Vis Sci 2012; 53: 8181-8185.

6 Chebbi CK, Kichenin K, Amar N, Nourry H, Warnet JM, Barritault D et al. Pilot study of a new matrix therapy agent (RGTA OTR4120) in treatment-resistant corneal ulcers and corneal dystrophy. J Fr Ophtalmol 2008; 31: 465-471.

7 Alexakis C, Mestries P, Garcia S, Petit E, Barbier V, PapyGarcia D et al. Structurally different RGTAs modulate collagen-type expression by cultured aortic smooth muscle cells via different pathways involving fibroblast growth factor-2 or transforming growth factor-beta1. FASEB J 2004; 18: 1147-1149.

8 Morvan FO, Baroukh B, Ledoux D, Caruelle JP, Barritault D, Godeau $\mathrm{G}$ et al. An engineered biopolymer prevents mucositis induced by 5 -fluorouracil in hamsters. Am J Pathol 2004; 164: 739-746.

9 Garcia-Filipe S, Barbier-Chassefiere V, Alexakis C, Huet E, Ledoux D, Kerros ME et al. RGTA OTR4120, a heparan sulfate mimetic, is a possible long-term active agent to heal burned skin. J Biomed Mater Res A 2007; 80: 75-84.

10 Araki-Sasaki K, Ohashi Y, Sasabe T, Hayashi K, Watanabe $\mathrm{H}$, Tano $\mathrm{Y}$ et al. An SV40-immortalized human corneal epithelial cell line and its characterization. Invest Ophthalmol Vis Sci 1995; 36: 614-621.

11 Katz S, Hukkanen M, Lounatmaa K, Rousselle P, Tervo T, Virtanen I. Cooperation of isoforms of laminin-332 and tenascin-CL during early adhesion and spreading of immortalized human corneal epithelial cells. Exp Eye Res 2006; 83: 1412-1422.

12 Greco D, Vellonen KS, Turner HC, Hakli M, Tervo T, Auvinen $\mathrm{P}$ et al. Gene expression analysis in SV-40 immortalized human corneal epithelial cells cultured with an air-liquid interface. Mol Vis 2010; 16: 2109-2120.

13 Liang CC, Park AY, Guan JL. In vitro scratch assay: a convenient and inexpensive method for analysis of cell migration in vitro. Nat Protoc 2007; 2: 329-333.

14 Robciuc A, Hyotylainen T, Jauhiainen M, Holopainen JM. Hyperosmolarity-induced lipid droplet formation depends on ceramide production by neutral sphingomyelinase 2 . J Lipid Res 2012; 53: 2286-2295. 
15 Hua X, Su Z, Deng R, Lin J, Li DQ, Pflugfelder SC. Effects of L-carnitine, erythritol and betaine on pro-inflammatory markers in primary human corneal epithelial cells exposed to hyperosmotic stress. Curr Eye Res 2015; 40: 657-667.

16 Hua X, Deng R, Li J, Chi W, Su Z, Lin J et al. Protective Effects of L-Carnitine Against Oxidative Injury by Hyperosmolarity in Human Corneal Epithelial Cells. Invest Ophthalmol Vis Sci 2015; 56: 5503-5511.

17 Deng R, Su Z, Hua X, Zhang Z, Li DQ, Pflugfelder SC. Osmoprotectants suppress the production and activity of matrix metalloproteinases induced by hyperosmolarity in primary human corneal epithelial cells. Mol Vis 2014; 20: 1243-1252.
18 Corrales RM, Luo L, Chang EY, Pflugfelder SC. Effects of osmoprotectants on hyperosmolar stress in cultured human corneal epithelial cells. Cornea 2008; 27: 574-579.

19 De Monchy I, Labbe A, Pogorzalek N, Gendron G, M'Garrech M, Kaswin G et al. Management of herpes zoster neurotrophic ulcer using a new matrix therapy agent (RGTA): A case report. J Fr Ophtalmol 2012; 35: 187 e16.

20 Arvola RP, Robciuc A, Holopainen JM. Matrix Regeneration Therapy: A Case Series of Corneal Neurotrophic Ulcers. Cornea 2016; 35: 451-455.

21 Kymionis GD, Liakopoulos DA, Grentzelos MA, Tsoulnaras KI, Detorakis ET, Cochener B et al. Effect of the regenerative agent poly(carboxymethylglucose sulfate) on corneal wound healing after corneal cross-linking for keratoconus. Cornea 2015; 34: 928-931. 